\title{
A case report of retroperitoneal Castleman disease
}

\author{
Peng Bo, MD; ${ }^{*}$ Zheng Junhua, MD; ${ }^{\dagger}$ Gao Qiruo, MSc ${ }^{\dagger}{ }^{\dagger}$ Li Hong, $M S c^{*}$
}

\begin{abstract}
Castleman disease (CD) is an uncommon lymphoproliferative disorder and is especially rare in the retroperitoneum or perirenal area. We report the case of a 42-year-old woman in whom we found on routine physical examination a mass localized in the right abdomen without clinically important constitutional symptoms for 30 days. Abdominal ultrasound, magnetic resonance images and computed tomography scans revealed a solid mass localized just below the right kidney in the retroperitoneum. The patient subsequently underwent an exploratory laparotomy. Pathological examination revealed a right retroperitoneal mass of $\mathrm{CD}$ (hyaline vascular type). The patient completely recovered after surgery. Castleman disease is commonly misdiagnosed as malignant lymphoma, lymphadenitis or ectopic thymoma. So far, its diagnosis is mainly achieved via histopathological examination of surgically obtained tissue. After removal of the lesion, local CD has a good prognosis.
\end{abstract}

Can Urol Assoc J 2009;3(3):E14-E16

\section{Résumé}

La maladie de Castleman est un trouble lymphoprolifératif rare, surtout dans les régions rétropéritonéale ou périrénale. Nous décrivons le cas d'une femme de 42 ans chez qui un examen physique de routine a révélé la présence d'une masse localisée au côté droit de l'abdomen sans que cette patiente n'affiche de symptômes constitutionnels d'importance clinique pendant 30 jours. Des images obtenues par échographie abdominale, par IRM et par tomodensitométrie ont révélé la présence d'une masse solide située juste en dessous du rein droit dans la région rétropéritonéale. La patiente a ensuite subi une laparotomie exploratoire. L'examen d'histopathologie a révélé une masse rétropéritonéale droite caractéristique de la maladie de Castleman dans sa forme hyaline vasculaire. La patiente s'est complètement rétablie après I'intervention. Au moment du diagnostic, la maladie de Castleman est souvent confondue avec un lymphome malin, une lymphadénite ou un thymome ectopique. Jusqu'à présent, le diagnostic de cette maladie est posé principalement sur la base d'un examen histopathologique de tissus prélevés par chirurgie. Après ablation de la masse, le pronostic lié à la maladie de Castleman est favorable.

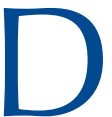

uring a routine physical examination, we found a 42-year old woman to have a nontender mass localized in the right abdomen for 30 days' duration. She had no systemic symptoms such as sweating, weight loss, fatigue or fever. The patient's history was not relevant, and she reported no marked surgical history or family history of cancer or disease. Physical examination revealed the presence of an $8 \times 7-\mathrm{cm}$ well-defined nontender mass with a smooth surface in the right abdomen. An abdominal ultrasound revealed a solid mass localized in the retroperitoneum with heterogeneous echo. A computed tomography (CT) scan of her abdomen before contrast medium administration showed a well-encapsulated mass of homogeneous hypodensity localized just below the right kidney in the retroperitoneum; the scan also showed obvious enhancement in the mass during the hepatic arterial phase. The density of the mass was similar to that of the aorta. It remained enhanced in the portal venous phase and delayed phase. An intravenous urogram (IVU) revealed that the mass was causing pressure to the right upper-middle ureter; her kidneys were normal. Magnetic resonance images (MRIs) showed that the mass measured $8.5 \times 7.5 \times 5.5 \mathrm{~cm}$ with clear margins and that it was positioned beneath the right kidney close to the inferior vena cava. The mass was intermediate in signal intensity on $T_{1}$-weighted images and highintensity on $T_{2}$-weighted images. Digital subtraction angiography showed that the mass received its blood supply from the right renal artery and the ureteral artery.

After bowel preparation, the patient underwent an exploratory laparotomy. The results revealed a well-defined mass densely adherent to the inferior vena cava in the right retroperitoneum. We excised the mass. Macroscopically, the surgical specimen was a well-circumscribed, encapsulated mass that measured $9 \times 7 \times 6 \mathrm{~cm}$. The cut surface was a dull red in colour and had a polycystic appearance. The histological examination showed a mass of lymphoid tissue with many follicles. These lymph node follicular dendritic cells had thickened and laminated mantle zones made of concentric rings of small lymphocytes (onion skin) surrounding small atrophic germinal centres (Fig. 1). The interfollicular areas were extremely vascular, and some vessels entered the abnormal germinal centres. We found no sinuses. We obtained positive immunohistochemistry results after staining for LCA, CD34, CD68, CD20, CD45 RO, SMA and S-100. Pathological examination revealed a right retroperitoneal mass, which we diagnosed as Castleman disease (CD; hyaline vascular type). The patient recovered without evidence of recurrence, and the follow-up examinations revealed no abnormality. 


\section{Discussion}

Although the etiology of $\mathrm{CD}$ remains unclear, the pathogenesis is related to an abnormal immune response to viral infections or medication side effects that can cause similar changes in hyperplastic reactive lymph nodes. ${ }^{1}$ Some studies found that inflammatory cytokines were associated with the manifestation of a variety of symptoms accompanying $C D$, especially for plasma cell type or multicentric $\mathrm{CD}$ (MCD). There are 3 types of CD: hyaline vascular type, plasma cell type and mixed type. ${ }^{2}$ Unicentric hyaline vascular CD is the most common histological form of the disease. Clinically, according to the involved range it can be divided into 2 types: MCD and local CD (LCD). ${ }^{2}$

The clinical features of LCD are painless enlarged lymph nodes, usually localized in the mediastinum, cervix, axillary lymph nodes or abdomen; it may also occur in the muscles, larynx and lungs and orbits where no lymph nodes exist. Some patients experience fever, sweat, fatigue, anorexia and substantial weight loss. Laboratory examinations reveal increased serum iron protein and inflammatory proteins of polyclonal hyperimmunoglobulinia and CRP, decreased serum iron and total iron-binding capacity and increased interleukin (IL)-6. Coombs test auto antibodies

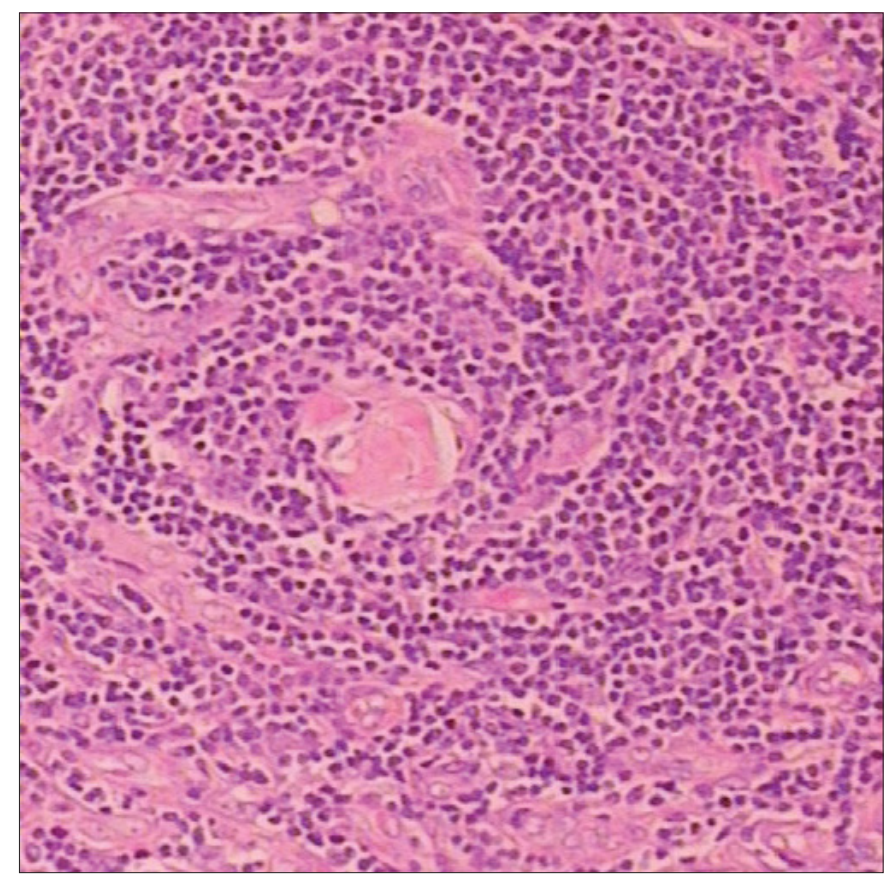

Fig. 1. A mass of lymphoid tissue with many follicles. These lymph node follicular dendritic cells had thickened and laminated mantle zones composed of concentric rings of small lymphocytes (onion skin) surrounding small atrophic germinal centres. The interfollicular areas were extremely vascular, and some vessels entered the abnormal germinal centres. We found no sinuses. Hematoxylin and eosin staining, original magnification $\times 400$. and rheumatoid factors are positive. Except for abnormal results of the laboratory tests mentioned previously, most patients with MCD experience inflammation caused by increased IL-6 and organ damage resulting from autoimmune abnormality. The hepatitis B and Epstein-Barr viruses and HIV are the result of the immune perturbation that may be seen with $\mathrm{CD}$ or, perhaps, forerunners of its evolution. ${ }^{3}$

Computed tomography scans not only show the locations of masses, but also demonstrate the forms and sizes of the masses. Castleman disease is characterized by the dendritic or mottling calcified lesions seen on CT scans. The effects of MRI are the same as CT, but radiographs are not helpful in diagnosing CD.

In patients with LCD, complete surgical resection is nearly always successful regardless of type. The surgical removal of involved lymph nodes can resolve local and systemic symptoms and is associated with a good prognosis. Unlike patients with $L C D$, symptomatic patients with $M C D$ require systemic therapy and several available alternatives matched to their needs and overall condition, including chemotherapy, steroids and antiviral therapy. Suppression of the immune response is the most important component of therapy. ${ }^{4}$ The use of prednisone or other steroids will frequently and promptly improve symptoms. In some serious cases, patients can take combination cyclophosphamide, vincristine and prednisone chemotherapy. Casquero and colleagues ${ }^{5}$ reported that MCD had been exploited therapeutically through the use of rituximab, a monoclonal antibody of CD20. Nishimoto and colleagues ${ }^{6}$ reported the use of cilizumab, a humanized monoclonal antibody of the human IL-6 receptor in a series of 28 HIV-negative patients. Recognition of the contribution of IL- 6 inhibition to the development of MCD has led to an attempt to block IL-6 or its effects directly. ${ }^{6}$ Long-term maintenance with IL-6 inhibition may be beneficial and has a good tolerance. The prognosis for patients with MCD is usually poor, especially among those with the plasma cell type, in whom the systemic disease follows a usually aggressive and often fatal course due to infectious complications, progressive disease and the association of cancerous tumours such as lymphoma or Kaposi sarcoma of several months' duration.

From the *Department of Urology, West China Hospital, Sichuan University, Chengdu, China and the †Department of Urology, Shanghai Tenth People's Hospital, Tongii University, Shanghai, China

This article has been peer reviewed.

Competing interests: None declared.

\section{References}

1. Sun H, Wang R, Bin H, et al. Localized Castleman disease with paraneoplastic pemphigus and pulmonary 
Bo et al.

involvement: clinical features and histopathology [article in Chinese]. Zhonghua Yi Xue Za Zhi. 2002;82:530-3.

2. Cobzeanu MD, Rusu D, Negru D, et al. Castleman disease of the neck. Rev Med Chir Soc Med Nat lasi 2005; 109:567-72.

3. Ning L, Fa-Bo Q, Feng DL. Epidemiological and clinical characteristics of Castleman's disease. World Chinese Journal of Digestology 2008;16:3469-73.

4. Fowler A, Collins L, de Ruiter A, et al. Multicentric Castleman's disease in a patient with HIV. Int J STD AIDS 2006;17:63-4.
5. Casquero A, Barroso A, Fernandez Guerrero ML, et al. Use of rituximab as a salvage therapy for HIVassociated multicentric Castleman disease. Ann Hematol 2006;85:185-7.

6. Nishimoto N, Kanakura Y, Aozasa K, et al. Humanized antiinterleukin-6 receptor antibody treatment of multicentric Castleman disease. Blood 2005;106:2627-32.

Correspondence: Dr. Zheng Junhua; №. 301 Middle Yanchang Rd, The Tenth Hospital, Shanghair, 200072; fax 008621-66303983; zhengih0471@sina.com 